Supporting Information

\title{
Conformational Interconversions of Amino Acid Derivatives
}

\section{Jakub Kaminský and Frank Jensen}

\section{Contents}

Figure S1. Correlations of MM $E_{\text {rel }}$ with the MP2 data $(\mathrm{kJ} / \mathrm{mol})$ for Ac-Ser-NHMe obtained using the sequential optimization procedure Table S1. Structural parameters and relative energies of Ac-Ser-NHMe transition states.

Table S2. Structural parameters and relative energies of Ac-Cys-NHMe transition states. 


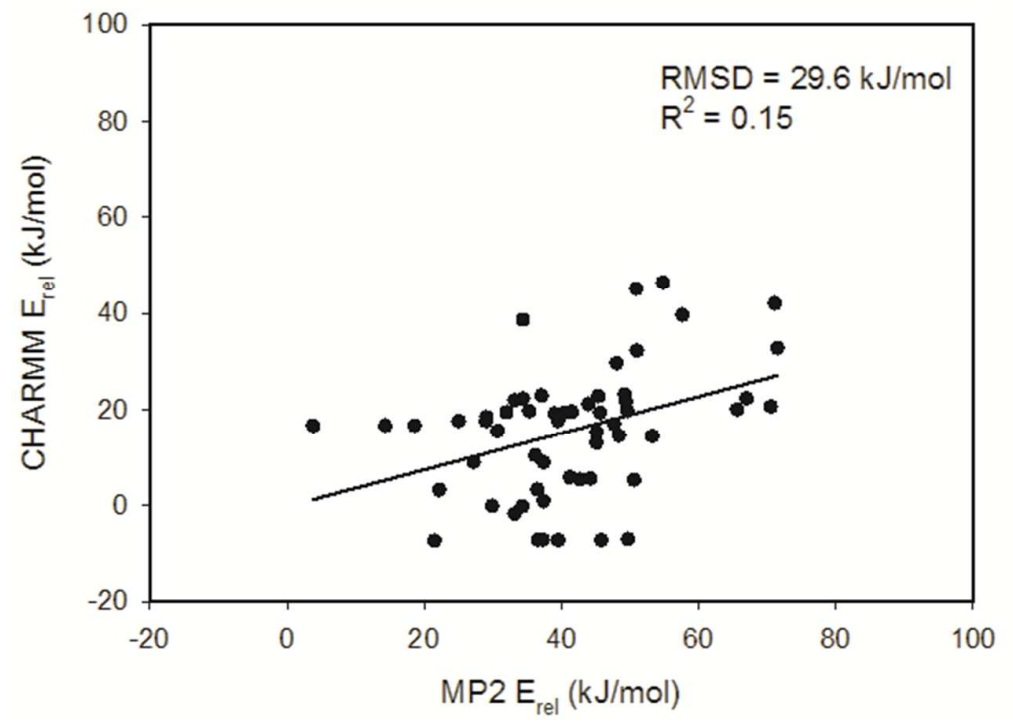

Figure S1. Correlations of the force field $E_{\text {rel }}$ with the MP2 data $(\mathrm{kJ} / \mathrm{mol})$ for transition states of Ac-Ser-NHMe obtained using the sequential optimization procedure (See Computational details for more info). The $R^{2}$ values indicate the square of the correlation coefficients. 
Journal of Chemical Theory and Computation

Table S1. Transition states for Ac-Ser-NHMe.

\begin{tabular}{|c|c|c|c|c|c|c|c|c|c|c|c|c|c|c|c|}
\hline TS & Reg. & Vibr. Mode & $\phi$ & $\psi$ & $\chi_{1}$ & $\chi_{2}$ & MP2 & AMBER & AMBER14SB & MM2 & MM3 & MMFFs & OPLS & CHARMM & AMOEBA \\
\hline 1 & $\alpha_{D}$ & $-\mathrm{OH}$ rotation & 63 & 36 & -54 & -117 & 3.8 & 37.6 & 37.4 & 29.8 & 36.9 & 25.8 & 51.8 & 29.9 & 30.5 \\
\hline 2 & $\alpha_{D}$ & $-\mathrm{OH}$ rotation & 70 & 25 & -104 & -83 & 14.3 & 47.6 & 49.3 & 38.3 & 40.6 & 34.9 & 50.0 & 48.0 & 40.3 \\
\hline 3 & $\alpha_{D}$ & $-\mathrm{OH}$ rotation & 69 & 25 & -49 & -5 & 18.6 & 47.8 & 53.2 & 41.2 & 48.2 & 40.9 & 56.9 & 27.6 & 40.9 \\
\hline 4 & $\beta_{\mathrm{L}}$ & $\mathrm{C} \alpha-\mathrm{CH}_{2} \mathrm{OH}$ torsion & -165 & 178 & -127 & 66 & 21.5 & 18.6 & 20.7 & 34.7 & 19.9 & 15.8 & 20.2 & 38.1 & 35.0 \\
\hline 5 & $\mathrm{C}_{7}^{\mathrm{eq}}$ & $-\mathrm{OH}$ rotation & -87 & 69 & -60 & -124 & 22.2 & 13.8 & 19.0 & 11.4 & 13.1 & 14.8 & 24.9 & 50.4 & 57.0 \\
\hline 6 & $\beta_{\mathrm{L}}$ & $-\mathrm{OH}$ rotation & -160 & 179 & 71 & -137 & 25.0 & 29.4 & 27.7 & 32.4 & 25.5 & 31.1 & 32.1 & 24.5 & 43.6 \\
\hline 7 & $\mathrm{C}_{7}^{\mathrm{eq}}$ & $-\mathrm{OH}$ rotation & -80 & 80 & -52 & -3 & 27.2 & 17.8 & 27.4 & 20.8 & 23.3 & 16.5 & 26.4 & 42.2 & 49.3 \\
\hline 8 & $\mathrm{C}_{7}{ }^{\mathrm{ax}}$ & $-\mathrm{OH}$ rotation & 81 & -61 & -54 & 128 & 29.0 & 21.3 & 30.3 & 15.4 & 16.2 & 24.3 & 37.7 & 19.5 & 33.0 \\
\hline 9 & $\mathrm{C}_{7}^{\text {ax }}$ & $\mathrm{C} \alpha-\mathrm{CH}_{2} \mathrm{OH}$ torsion & 71 & -22 & -160 & -44 & 29.1 & 16.8 & 15.9 & 11.6 & 14.5 & 32.3 & 25.8 & 46.1 & 54.7 \\
\hline 10 & $\mathrm{C}_{7}{ }^{\mathrm{eq}}$ & $-\mathrm{OH}$ rotation & -87 & 72 & -5 & 140 & 29.9 & 29.6 & 37.1 & 23.8 & 21.1 & 24.9 & 38.3 & 36.3 & 48.7 \\
\hline 11 & $\alpha_{\mathrm{L}}$ & -OH rotation & -76 & -21 & -51 & -130 & 30.7 & 19.6 & 29.1 & 26.7 & 23.0 & 23.4 & 34.8 & 39.0 & 56.3 \\
\hline 12 & $\mathrm{C}_{7}^{\mathrm{ax}}$ & amide torsion torsion & 74 & -60 & -168 & -61 & 32.0 & 25.3 & 24.0 & 11.4 & 15.3 & 36.0 & 27.8 & 40.1 & 51.5 \\
\hline 13 & $\beta_{\mathrm{L}}$ & amide torsion torsion & 178 & -117 & 57 & -67 & 33.2 & 35.0 & 39.8 & 41.3 & 12.7 & 25.0 & 26.3 & 20.4 & 37.5 \\
\hline 14 & $\mathrm{C}_{7}^{\text {eq }}$ & amide torsion torsion & -98 & 118 & 180 & 71 & 33.2 & 42.0 & 34.4 & 33.8 & 29.6 & 45.9 & 32.8 & 42.3 & 53.9 \\
\hline 15 & $\mathrm{C}_{7}^{\mathrm{eq}}$ & $\mathrm{C} \alpha-\mathrm{CH}_{2} \mathrm{OH}$ torsion & -84 & 70 & 13 & -89 & 34.3 & 37.7 & 46.1 & 26.1 & 27.7 & 29.6 & 36.9 & 39.2 & 51.6 \\
\hline 16 & $\delta_{\mathrm{L}}$ & amide torsion torsion & -154 & 78 & -47 & -47 & 34.4 & 26.9 & 33.1 & 31.6 & 22.4 & 28.1 & 39.7 & 47.0 & 59.3 \\
\hline 17 & $\alpha_{D}$ & $\mathrm{C} \alpha-\mathrm{CH}_{2} \mathrm{OH}$ torsion & 56 & 41 & 22 & -80 & 34.4 & 84.5 & 83.9 & 54.8 & 75.5 & 73.1 & 95.8 & 18.0 & 21.4 \\
\hline 18 & $\varepsilon_{\mathrm{L}}$ & $-\mathrm{OH}$ rotation & -70 & 166 & 56 & 136 & 35.3 & 42.7 & 41.3 & 37.4 & 44.1 & 37.4 & 46.6 & 47.7 & 49.3 \\
\hline 19 & $\mathrm{C}_{7}{ }^{\mathrm{eq}}$ & $-\mathrm{OH}$ rotation & -81 & 92 & -175 & 6 & 36.2 & 41.5 & 43.4 & 27.9 & 30.3 & 44.1 & 42.2 & 35.1 & 47.0 \\
\hline 20 & $\mathrm{C}_{7}{ }^{\mathrm{eq}}$ & $\mathrm{C} \alpha-\mathrm{CH}_{2} \mathrm{OH}$ torsion & -84 & 76 & -117 & -167 & 36.5 & 35.2 & 42.0 & 25.3 & 27.9 & 30.4 & 45.8 & 32.2 & 47.3 \\
\hline 21 & $\beta_{\mathrm{L}}$ & $\mathrm{C} \alpha-\mathrm{CH}_{2} \mathrm{OH}$ torsion & -169 & 141 & 123 & -63 & 36.6 & 46.4 & 36.0 & 45.8 & 24.5 & 54.4 & 38.9 & 43.1 & 57.1 \\
\hline 22 & $\mathrm{C}_{7}^{\mathrm{ax}}$ & $-\mathrm{OH}$ rotation & 64 & -29 & 52 & 23 & 37.1 & 19.8 & 24.7 & 17.0 & 25.7 & 29.9 & 36.3 & 18.2 & 35.8 \\
\hline 23 & $\beta_{\mathrm{L}}$ & $-\mathrm{OH}$ rotation & -149 & 141 & 172 & 14 & 37.3 & 48.7 & 42.2 & 47.8 & 32.7 & 56.9 & 39.7 & 51.8 & 66.2 \\
\hline 24 & $\mathrm{C}_{7}^{\mathrm{eq}}$ & $-\mathrm{OH}$ rotation & -80 & 93 & 114 & -22 & 37.4 & 39.2 & 41.5 & 31.3 & 32.7 & 46.6 & 47.5 & 44.2 & 56.2 \\
\hline 25 & $\mathrm{C}_{7}{ }^{\mathrm{eq}}$ & $-\mathrm{OH}$ rotation & -85 & 73 & -113 & -80 & 37.4 & 35.5 & 40.3 & 27.3 & 29.9 & 32.8 & 40.3 & 41.4 & 40.8 \\
\hline 26 & $\mathrm{C}_{7}^{\mathrm{eq}}$ & $\mathrm{C} \alpha-\mathrm{CH}_{2} \mathrm{OH}$ torsion & -83 & 79 & -123 & 68 & 39.0 & 40.0 & 44.5 & 31.2 & 35.0 & 32.9 & 42.2 & 31.9 & 31.3 \\
\hline 27 & $\beta_{\mathrm{L}}$ & -OH rotation & -161 & -176 & 118 & -176 & 39.5 & 43.6 & 42.5 & 38.5 & 28.8 & 40.2 & 42.4 & 35.4 & 31.9 \\
\hline 28 & $\mathrm{C}_{7}^{\mathrm{ax}}$ & $-\mathrm{OH}$ rotation & 74 & -66 & -50 & -8 & 39.5 & 30.7 & 41.7 & 25.5 & 33.0 & 32.3 & 46.5 & 36.7 & 45.4 \\
\hline 29 & $\mathrm{C}_{7}{ }^{\mathrm{ax}}$ & $-\mathrm{OH}$ rotation & 75 & -43 & -107 & -72 & 40.3 & 32.6 & 38.4 & 25.7 & 28.4 & 32.5 & 38.7 & 50.2 & 60.0 \\
\hline 30 & $\alpha_{\mathrm{L}}$ & $-\mathrm{OH}$ rotation & -98 & -4 & 133 & 41 & 41.2 & 42.6 & 46.0 & 31.5 & 35.2 & 64.8 & 53.3 & 18.3 & 30.1 \\
\hline 31 & $\mathrm{C}_{7}^{\mathrm{ax}}$ & $\mathrm{C} \alpha-\mathrm{CH}_{2} \mathrm{OH}$ torsion & 70 & -43 & 13 & 61 & 41.5 & 26.1 & 31.3 & 21.2 & 28.0 & 32.1 & 44.9 & 27.8 & 36.9 \\
\hline 32 & $\mathrm{C}_{7}^{\mathrm{eq}}$ & -OH rotation & -106 & 16 & -4 & 170 & 42.7 & 31.4 & 43.1 & 31.0 & 24.3 & 30.1 & 41.4 & 48.0 & 59.5 \\
\hline
\end{tabular}


Journal of Chemical Theory and Computation

\begin{tabular}{|c|c|c|c|c|c|c|c|c|c|c|c|c|c|c|c|}
\hline 33 & $\mathrm{C}_{7}^{\mathrm{ax}}$ & $\mathrm{C} \alpha-\mathrm{CH}_{2} \mathrm{OH}$ torsion & 76 & -71 & -117 & -176 & 43.9 & 37.6 & 47.1 & 22.9 & 26.1 & 31.6 & 49.1 & 81.4 & 84.6 \\
\hline 34 & $\mathrm{C}_{7}{ }^{\mathrm{eq}}$ & -OH rotation & -80 & 26 & 66 & -60 & 44.2 & 41.8 & 42.5 & 25.3 & 39.1 & 40.2 & 40.7 & 35.5 & 46.7 \\
\hline 35 & $\mathrm{C}_{7}^{\text {eq }}$ & $-\mathrm{OH}$ rotation & -98 & 4 & -109 & -73 & 45.1 & 40.3 & 49.8 & 37.3 & 36.6 & 45.7 & 47.7 & 30.8 & 48.5 \\
\hline 36 & $\beta_{\mathrm{L}}$ & $-\mathrm{OH}$ rotation & -120 & 163 & -1 & -13 & 45.1 & 48.2 & 57.0 & 56.0 & 51.4 & 39.4 & 53.9 & 53.6 & 52.8 \\
\hline 37 & $\alpha_{D}$ & $\mathrm{C} \alpha-\mathrm{CH}_{2} \mathrm{OH}$ torsion & 69 & 31 & -3 & 83 & 45.4 & 46.4 & 46.7 & 34.9 & 39.5 & 34.1 & 59.3 & 25.9 & 48.7 \\
\hline 38 & $\mathrm{C}_{7}^{\mathrm{ax}}$ & $-\mathrm{OH}$ rotation & 76 & -56 & 132 & -63 & 45.6 & 48.9 & 46.7 & 25.4 & 36.2 & 62.0 & 50.9 & 82.2 & 91.8 \\
\hline 39 & $\beta_{\mathrm{L}}$ & $-\mathrm{OH}$ rotation & -165 & 156 & 128 & 56 & 45.8 & 61.0 & 52.3 & 53.5 & 40.8 & 64.3 & 50.5 & 56.6 & 66.1 \\
\hline 40 & $\alpha_{D}$ & amide torsion torsion & 8 & 82 & -55 & 171 & 47.7 & 36.5 & 48.9 & 42.1 & 52.8 & 66.1 & 59.8 & 83.7 & 86.2 \\
\hline 41 & $\mathrm{C}_{7}{ }^{\mathrm{ax}}$ & $-\mathrm{OH}$ rotation & 74 & -74 & -120 & 74 & 48.0 & 43.1 & 49.7 & 29.2 & 35.2 & 36.9 & 48.1 & 48.2 & 64.9 \\
\hline 42 & $\varepsilon_{\mathrm{D}}$ & $\mathrm{C} \alpha-\mathrm{CH}_{2} \mathrm{OH}$ torsion & 56 & -173 & 157 & -89 & 48.4 & 59.0 & 49.8 & 44.4 & 48.9 & 64.9 & 63.8 & 47.7 & 63.1 \\
\hline 43 & $\mathrm{C}_{7}^{\mathrm{ax}}$ & -OH rotation & 58 & -22 & 68 & 133 & 49.2 & 39.7 & 41.6 & 24.8 & 41.9 & 54.4 & 60.5 & 32.1 & 54.6 \\
\hline 44 & $\delta_{\mathrm{D}}$ & $-\mathrm{OH}$ rotation & -167 & -33 & 169 & 10 & 49.4 & 47.5 & 47.7 & 43.5 & 38.3 & 58.1 & 52.8 & 73.8 & 78.0 \\
\hline 45 & $\varepsilon_{\mathrm{D}}$ & $-\mathrm{OH}$ rotation & 37 & -119 & 67 & -133 & 49.6 & 56.9 & 61.0 & 42.3 & 53.2 & 73.2 & 79.0 & 52.6 & 64.6 \\
\hline 46 & $\beta_{\mathrm{L}}$ & $-\mathrm{OH}$ rotation & -155 & -159 & -151 & -76 & 49.7 & 61.5 & 59.8 & 59.6 & 56.7 & 65.4 & 52.5 & 44.4 & 41.0 \\
\hline 47 & $\beta_{\mathrm{L}}$ & $-\mathrm{OH}$ rotation & -112 & 146 & -113 & 75 & 50.6 & 54.4 & 38.6 & 51.3 & 44.1 & 47.5 & 52.6 & 10.7 & 27.2 \\
\hline 48 & $\mathrm{C}_{7}{ }^{\mathrm{ax}}$ & $-\mathrm{OH}$ rotation & 64 & -41 & 79 & -145 & 50.9 & 41.3 & 41.9 & 20.3 & 37.2 & 53.6 & 58.2 & 76.2 & 80.7 \\
\hline 49 & $\delta_{\mathrm{D}}$ & $\mathrm{C} \alpha-\mathrm{CH}_{2} \mathrm{OH}$ torsion & -166 & -45 & -9 & -62 & 51.0 & 36.6 & 45.0 & 42.7 & 34.3 & 37.0 & 50.5 & 49.3 & 63.5 \\
\hline 50 & $\varepsilon_{\mathrm{D}}$ & $\mathrm{C} \alpha-\mathrm{CH}_{2} \mathrm{OH}$ torsion & 59 & -174 & -104 & 73 & 53.2 & 55.3 & 57.1 & 59.1 & 64.7 & 50.5 & 64.3 & 27.4 & 35.8 \\
\hline 51 & $\alpha_{\mathrm{L}}$ & $-\mathrm{OH}$ rotation & -65 & -40 & 174 & -145 & 54.8 & 60.5 & 64.0 & 36.2 & 50.2 & 71.5 & 69.7 & 40.1 & 39.8 \\
\hline 52 & $\alpha_{D}$ & amide torsion torsion & 85 & 109 & -175 & -95 & 57.6 & 63.2 & 59.1 & 56.7 & 41.7 & 68.7 & 54.5 & 11.7 & 14.9 \\
\hline 53 & $\mathrm{C}_{7}{ }^{\mathrm{ax}}$ & $-\mathrm{OH}$ rotation & 58 & -75 & 13 & -54 & 65.6 & 52.3 & 61.1 & 38.5 & 50.4 & 55.9 & 73.3 & 37.9 & 38.9 \\
\hline 54 & $\delta_{\mathrm{D}}$ & $-\mathrm{OH}$ rotation & -168 & -36 & 118 & -72 & 67.0 & 76.4 & 75.7 & 59.7 & 57.2 & 79.0 & 72.0 & 24.1 & 32.1 \\
\hline 55 & $\delta_{\mathrm{D}}$ & $-\mathrm{OH}$ rotation & -142 & -48 & -9 & 19 & 70.5 & 66.4 & 80.4 & 66.9 & 65.8 & 63.6 & 76.8 & 64.9 & 71.4 \\
\hline 56 & $\delta_{\mathrm{D}}$ & $\mathrm{C} \alpha-\mathrm{CH} 2 \mathrm{OH}$ torsion & 166 & -28 & -100 & -76 & 71.1 & 77.4 & 90.2 & 61.6 & 54.1 & 69.9 & 82.3 & 41.0 & 54.0 \\
\hline 57 & $\delta_{\mathrm{D}}$ & $-\mathrm{OH}$ rotation & -164 & -37 & -101 & -64 & 71.5 & 66.2 & 73.2 & 57.5 & 54.6 & 62.9 & 73.8 & 51.2 & 60.0 \\
\hline \multirow{2}{*}{\multicolumn{2}{|c|}{$\begin{array}{l}\mathbf{R}^{2} \\
\mathbf{R}^{2} \square\end{array}$}} & & & & & & & 12.6 & 12.2 & 13.8 & 13.4 & 12.6 & 14.6 & 11.5 & 14.8 \\
\hline & & & & & & & & 0.40 & 0.48 & 0.31 & 0.34 & 0.48 & 0.42 & 0.52 & 0.49 \\
\hline
\end{tabular}


Journal of Chemical Theory and Computation

Table S2. Transition states for Ac-Cys-NHMe.

\begin{tabular}{|c|c|c|c|c|c|c|c|c|c|c|c|c|c|c|c|}
\hline TS & Reg. & Vibr. Mode & $\phi$ & $\psi$ & $\chi_{1}$ & $\chi_{2}$ & MP2 & AMBER & AMBER14SB & MM2 & MM3 & MMFFs & OPLS & CHARMM & АМОЕВА \\
\hline 1 & $\beta_{\mathrm{L}}$ & $\mathrm{C} \alpha-\mathrm{CH}_{2} \mathrm{SH}$ torsion & -164 & 167 & 61 & 67 & 13.6 & 5.3 & 13.8 & 26.2 & 5.3 & 16.3 & 14.9 & 6.0 & 17.3 \\
\hline 2 & $\mathrm{C}_{7}{ }^{\mathrm{eq}}$ & $-\mathrm{SH}$ rotation & -88 & 78 & -59 & 113 & 15.1 & 10.9 & 11.3 & 8.6 & 5.6 & 11.7 & 24.6 & 11.8 & 13.3 \\
\hline 3 & $\mathrm{C}_{7}{ }^{\mathrm{eq}}$ & -SH rotation & -86 & 76 & -61 & -113 & 15.6 & 9.5 & 7.0 & 5.2 & 3.0 & 9.1 & 21.2 & 8.5 & 13.0 \\
\hline 4 & $\mathrm{C}_{7}{ }^{\mathrm{eq}}$ & -SH rotation & -83 & 80 & -58 & 0 & 16.6 & 6.2 & 13.9 & 11.3 & 6.5 & 7.5 & 20.1 & 10.6 & 13.3 \\
\hline 5 & $\mathrm{C}_{7}{ }^{\mathrm{eq}}$ & amide torsion & -114 & 38 & 60 & -109 & 18.1 & 25.4 & 29.2 & 16.9 & 12.6 & 22.9 & 26.1 & 24.8 & 25.3 \\
\hline 6 & $\beta_{\mathrm{L}}$ & -SH rotation & -165 & 168 & 63 & -132 & 19.8 & 11.0 & 20.4 & 29.3 & 10.8 & 22.2 & 21.3 & 10.7 & 22.0 \\
\hline 7 & $\beta_{\mathrm{L}}$ & -SH rotation & -156 & 178 & 58 & 130 & 19.9 & 17.1 & 23.3 & 31.0 & 16.1 & 25.3 & 23.8 & 13.9 & 29.4 \\
\hline 8 & $\mathrm{C}_{7}{ }^{\mathrm{eq}}$ & $\mathrm{C} \alpha-\mathrm{CH}_{2} \mathrm{SH}$ torsion & -85 & 72 & 7 & -76 & 20.2 & 27.6 & 29.3 & 26.4 & 22.9 & 24.6 & 25.1 & 30.2 & 24.4 \\
\hline 9 & $\mathrm{C}_{7}^{\mathrm{ax}}$ & amide torsion & 74 & -58 & -165 & -48 & 21.1 & 14.4 & 11.4 & 5.5 & 2.3 & 22.2 & 17.6 & 11.8 & 25.2 \\
\hline 10 & $\mathrm{C}_{7}^{\mathrm{ax}}$ & -SH rotation & 75 & -62 & -57 & -122 & 21.4 & 17.9 & 17.3 & 7.8 & 8.5 & 17.0 & 32.9 & 16.0 & 29.5 \\
\hline 11 & $\mathrm{C}_{7}^{\mathrm{ax}}$ & -SH rotation & 79 & -63 & -62 & 148 & 21.6 & 15.0 & 17.4 & 6.5 & 3.7 & 14.9 & 29.5 & 12.1 & 29.2 \\
\hline 12 & $\mathrm{C}_{7}^{\mathrm{eq}}$ & $\mathrm{C} \alpha-\mathrm{CH}_{2} \mathrm{SH}$ torsion & -87 & 75 & -12 & 95 & 21.9 & 28.3 & 24.0 & 26.4 & 24.1 & 27.8 & 31.7 & 30.2 & 25.3 \\
\hline 13 & $\mathrm{C}_{7}{ }^{\mathrm{eq}}$ & -SH rotation & -83 & 45 & 53 & 149 & 22.4 & 14.6 & 8.9 & 3.1 & 3.8 & 7.4 & 10.8 & 8.5 & 32.6 \\
\hline 14 & $\mathrm{C}_{7}{ }^{\text {eq }}$ & -SH rotation & -82 & 91 & -172 & 9 & 22.8 & 21.3 & 30.1 & 21.9 & 13.7 & 26.2 & 25.2 & 21.9 & 21.8 \\
\hline 15 & $\delta_{\mathrm{L}}$ & amide torsion & -152 & 63 & -171 & -71 & 23.0 & 12.6 & 20.5 & 22.6 & 6.1 & 28.2 & 21.3 & 17.8 & 24.7 \\
\hline 16 & $\beta_{\mathrm{L}}$ & amide torsion & -120 & 139 & -60 & -171 & 25.8 & 12.5 & 23.0 & 29.6 & 10.1 & 27.8 & 35.1 & 10.6 & 19.7 \\
\hline 17 & $\mathrm{C}_{7}^{\mathrm{eq}}$ & $\mathrm{C} \alpha-\mathrm{CH}_{2} \mathrm{SH}$ torsion & -84 & 73 & -113 & -72 & 26.6 & 24.3 & 23.9 & 21.1 & 16.9 & 25.5 & 28.7 & 27.4 & 25.9 \\
\hline 18 & $\mathrm{C}_{7}^{\mathrm{eq}}$ & -SH rotation & -118 & 17 & -58 & -121 & 26.7 & 23.0 & 24.1 & 19.8 & 13.1 & 26.1 & 38.3 & 14.1 & 34.4 \\
\hline 19 & $\alpha_{D}$ & -SH rotation & 65 & 33 & -56 & -125 & 26.8 & 35.4 & 25.9 & 17.4 & 21.2 & 16.9 & 43.0 & 22.8 & 31.4 \\
\hline 20 & $\alpha_{D}$ & -SH rotation & 66 & 34 & -61 & 138 & 27.4 & 33.3 & 25.1 & 16.6 & 17.9 & 15.7 & 40.1 & 20.1 & 31.3 \\
\hline 21 & $\mathrm{C}_{7}^{\mathrm{ax}}$ & -SH rotation & 76 & -66 & -58 & -2 & 27.7 & 16.7 & 28.4 & 15.3 & 13.8 & 20.0 & 34.4 & 20.9 & 36.3 \\
\hline 22 & $\beta_{\mathrm{L}}$ & $\mathrm{C} \alpha-\mathrm{CH}_{2} \mathrm{SH}$ torsion & -162 & 148 & 119 & -55 & 28.7 & 20.8 & 27.5 & 43.1 & 18.2 & 44.1 & 32.6 & 32.0 & 33.1 \\
\hline 23 & $\mathrm{C}_{7}^{\mathrm{ax}}$ & -SH rotation & 74 & -73 & -171 & -138 & 28.8 & 24.0 & 22.2 & 10.8 & 10.2 & 28.1 & 30.8 & 19.5 & 35.4 \\
\hline 24 & $\mathrm{C}_{7}{ }^{\mathrm{eq}}$ & $\mathrm{C} \alpha-\mathrm{CH}_{2} \mathrm{SH}$ torsion & -84 & 74 & -116 & -168 & 29.2 & 25.7 & 28.2 & 23.2 & 17.5 & 28.9 & 32.1 & 28.4 & 29.0 \\
\hline 25 & $\beta_{\mathrm{L}}$ & $\mathrm{C} \alpha-\mathrm{CH}_{2} \mathrm{SH}$ torsion & -164 & 156 & 1 & -68 & 30.0 & 14.8 & 32.7 & 47.8 & 23.8 & 35.5 & 38.0 & 28.2 & 38.1 \\
\hline
\end{tabular}


Journal of Chemical Theory and Computation

\begin{tabular}{|c|c|c|c|c|c|c|c|c|c|c|c|c|c|c|c|}
\hline 26 & $\alpha_{\mathrm{L}}$ & -SH rotation & -83 & -9 & 66 & -27 & 30.8 & 32.9 & 30.6 & 28.0 & 24.1 & 34.7 & 28.2 & 25.6 & 39.8 \\
\hline 27 & $\mathrm{C}_{7}^{\mathrm{ax}}$ & -SH rotation & 72 & -76 & -178 & 139 & 31.0 & 26.3 & 23.8 & 13.0 & 13.1 & 32.1 & 35.0 & 23.1 & 31.1 \\
\hline 28 & $\mathrm{C}_{7}^{\mathrm{ax}}$ & $\mathrm{C} \alpha-\mathrm{CH}_{2} \mathrm{SH}$ torsion & 75 & -49 & -113 & -64 & 31.9 & 27.5 & 26.4 & 16.8 & 14.3 & 28.2 & 27.5 & 28.0 & 42.1 \\
\hline 29 & $\delta_{\mathrm{D}}$ & amide torsion & -151 & -35 & 60 & 84 & 32.1 & 35.8 & 26.0 & 27.6 & 21.3 & 28.8 & 26.3 & 31.6 & 40.2 \\
\hline 30 & $\beta_{\mathrm{L}}$ & amide torsion & -157 & 164 & -105 & -146 & 33.3 & 23.9 & 38.3 & 46.6 & 22.8 & 40.7 & 40.3 & 25.8 & 32.8 \\
\hline 31 & $\delta_{\mathrm{L}}$ & $\mathrm{C} \alpha-\mathrm{CH}_{2} \mathrm{SH}$ torsion & -157 & 31 & 3 & -73 & 34.3 & 30.6 & 42.1 & 40.1 & 27.1 & 33.3 & 42.2 & 39.7 & 50.5 \\
\hline 32 & $\beta_{\mathrm{L}}$ & $\mathrm{C} \alpha-\mathrm{CH}_{2} \mathrm{SH}$ torsion & -151 & 150 & -8 & 76 & 34.7 & 22.4 & 39.0 & 49.2 & 26.3 & 43.7 & 44.7 & 33.6 & 39.9 \\
\hline 34 & $\mathrm{C}_{7}^{\mathrm{ax}}$ & -SH rotation & 77 & -35 & 98 & 105 & 35.7 & 40.3 & 46.0 & 25.9 & 27.9 & 52.4 & 47.7 & 43.1 & 50.7 \\
\hline 35 & $\alpha_{\mathrm{D}}$ & -SH rotation & 67 & 31 & -62 & 2 & 35.8 & 37.1 & 40.9 & 29.1 & 29.8 & 27.1 & 46.0 & 31.8 & 41.5 \\
\hline 36 & $\mathrm{C}_{7}^{\text {ax }}$ & -SH rotation & 77 & -35 & 98 & 105 & 36.2 & 40.3 & 43.2 & 25.9 & 27.9 & 52.3 & 47.8 & 43.1 & 50.7 \\
\hline 37 & $\beta_{\mathrm{L}}$ & $\mathrm{C} \alpha-\mathrm{CH}_{2} \mathrm{SH}$ torsion & -160 & 150 & 124 & 63 & 36.4 & 29.1 & 37.4 & 49.6 & 24.6 & 51.7 & 39.4 & 37.4 & 42.7 \\
\hline 38 & $\alpha_{D}$ & $\mathrm{C} \alpha-\mathrm{CH}_{2} \mathrm{SH}$ torsion & 68 & 29 & -112 & -72 & 36.4 & 46.9 & 39.8 & 29.0 & 29.3 & 32.7 & 41.5 & 37.7 & 46.0 \\
\hline 39 & $\mathrm{C}_{7}^{\mathrm{ax}}$ & $\mathrm{C} \alpha-\mathrm{CH}_{2} \mathrm{SH}$ torsion & 76 & -48 & 135 & -75 & 37.3 & 35.9 & 35.3 & 23.9 & 24.4 & 52.4 & 45.6 & 43.1 & 54.1 \\
\hline 40 & $\mathrm{C}_{7}^{\mathrm{ax}}$ & $\mathrm{C} \alpha-\mathrm{CH}_{2} \mathrm{SH}$ torsion & 74 & -74 & -121 & 69 & 37.4 & 30.0 & 35.0 & 21.6 & 20.0 & 30.8 & 34.7 & 32.5 & 48.3 \\
\hline 42 & $\mathrm{C}_{7}^{\mathrm{eq}}$ & $\mathrm{C} \alpha-\mathrm{CH}_{2} \mathrm{SH}$ torsion & -112 & 18 & -112 & -69 & 38.4 & 34.7 & 38.1 & 31.9 & 22.9 & 40.1 & 39.8 & 29.3 & 44.0 \\
\hline 43 & $\mathrm{C}_{7}^{\mathrm{eq}}$ & $\mathrm{C} \alpha-\mathrm{CH}_{2} \mathrm{SH}$ torsion & -112 & 18 & -112 & -69 & 38.4 & 34.7 & 38.2 & 32.0 & 22.9 & 40.1 & 39.8 & 29.3 & 44.0 \\
\hline 44 & $\varepsilon_{\mathrm{L}}$ & $\mathrm{C} \alpha-\mathrm{CH}_{2} \mathrm{SH}$ torsion & -112 & 148 & -113 & -71 & 38.4 & 33.8 & 35.7 & 43.0 & 27.5 & 37.2 & 37.6 & 31.2 & 35.5 \\
\hline 45 & $\mathrm{C}_{7}^{\mathrm{ax}}$ & $\mathrm{C} \alpha-\mathrm{CH}_{2} \mathrm{SH}$ torsion & 75 & -72 & -119 & 180 & 39.0 & 29.4 & 35.5 & 20.3 & 16.4 & 31.4 & 35.4 & 29.8 & 47.7 \\
\hline 46 & $\beta_{\mathrm{L}}$ & $\mathrm{C} \alpha-\mathrm{CH}_{2} \mathrm{SH}$ torsion & -139 & 159 & -1 & -179 & 39.5 & 29.1 & 42.5 & 47.7 & 28.4 & 45.3 & 44.3 & 33.2 & 41.0 \\
\hline 47 & $\alpha_{L}$ & amide torsion & 5 & -16 & -56 & -74 & 40.1 & 51.3 & 34.5 & 36.5 & 52.2 & 62.1 & 44.5 & 45.3 & 45.1 \\
\hline 48 & $\mathrm{C}_{7}^{\text {ax }}$ & amide torsion & 64 & -25 & 93 & 75 & 40.3 & 33.3 & 30.3 & 19.2 & 22.2 & 44.1 & 42.9 & 32.2 & 48.5 \\
\hline 49 & $\delta_{\mathrm{D}}$ & -SH rotation & -160 & -46 & 176 & 7 & 40.6 & 36.9 & 38.1 & 41.4 & 28.2 & 45.7 & 42.0 & 41.6 & 45.8 \\
\hline 50 & $\alpha_{\mathrm{D}}$ & $\mathrm{C} \alpha-\mathrm{CH}_{2} \mathrm{SH}$ torsion & 65 & 36 & -123 & 72 & 40.7 & 52.3 & 51.1 & 35.6 & 36.5 & 43.3 & 49.1 & 43.9 & 51.8 \\
\hline 51 & $\mathrm{C}_{7}^{\text {ax }}$ & $\mathrm{C} \alpha-\mathrm{CH}_{2} \mathrm{SH}$ torsion & 64 & -31 & 16 & 61 & 42.5 & 33.8 & 29.2 & 29.2 & 34.9 & 41.9 & 46.7 & 38.9 & 52.5 \\
\hline 52 & $\delta_{\mathrm{D}}$ & amide torsion & -127 & -98 & -58 & -24 & 43.3 & 34.4 & 46.5 & 50.4 & 38.5 & 55.6 & 60.4 & 37.0 & 51.4 \\
\hline
\end{tabular}


Journal of Chemical Theory and Computation

\begin{tabular}{|c|c|c|c|c|c|c|c|c|c|c|c|c|c|c|c|}
\hline 53 & $\varepsilon_{\mathrm{D}}$ & amide torsion & 116 & 174 & -76 & 77 & 43.4 & 34.6 & 63.0 & 42.8 & 38.4 & 40.1 & 50.8 & 36.8 & 50.8 \\
\hline 54 & $\alpha_{D}$ & -SH rotation & 62 & 42 & -148 & 180 & 44.5 & 44.0 & 44.2 & 26.5 & 27.0 & 40.9 & 45.2 & 30.9 & 47.7 \\
\hline 55 & $\delta_{\mathrm{D}}$ & -SH rotation & -156 & -58 & 180 & -127 & 44.9 & 44.6 & 44.9 & 41.7 & 29.1 & 55.2 & 49.8 & 43.0 & 54.0 \\
\hline 56 & $\varepsilon_{\mathrm{D}}$ & $\mathrm{C} \alpha-\mathrm{CH}_{2} \mathrm{SH}$ torsion & 58 & -172 & -106 & 63 & 45.4 & 57.1 & 47.2 & 51.1 & 53.4 & 49.6 & 58.1 & 44.8 & 59.2 \\
\hline 57 & $\delta_{\mathrm{D}}$ & $\mathrm{C} \alpha-\mathrm{CH}_{2} \mathrm{SH}$ torsion & -167 & -32 & 122 & 57 & 46.7 & 48.2 & 47.8 & 49.9 & 36.7 & 54.7 & 53.8 & 64.5 & 60.8 \\
\hline 58 & $\delta_{\mathrm{D}}$ & $\mathrm{C} \alpha-\mathrm{CH}_{2} \mathrm{SH}$ torsion & -165 & -42 & -138 & 68 & 47.0 & 37.6 & 45.8 & 43.0 & 28.4 & 43.4 & 44.1 & 46.5 & 59.0 \\
\hline 59 & $\alpha_{\mathrm{L}}$ & amide torsion & -98 & -49 & -65 & 47 & 47.3 & 41.6 & 41.5 & 34.9 & 32.9 & 45.4 & 52.0 & 36.1 & 45.1 \\
\hline 60 & $\delta_{\mathrm{D}}$ & amide torsion & -157 & -49 & -98 & 47 & 47.4 & 30.3 & 39.2 & 49.1 & 35.7 & 39.4 & 45.7 & 41.8 & 55.7 \\
\hline 61 & $\alpha_{\mathrm{D}}$ & amide torsion & 55 & 23 & 59 & -177 & 47.5 & 46.1 & 51.0 & 30.2 & 37.9 & 49.7 & 62.4 & 45.9 & 54.6 \\
\hline 62 & $\alpha_{L}$ & -SH rotation & -65 & -41 & -172 & -134 & 47.5 & 52.8 & 44.8 & 38.5 & 38.9 & 60.1 & 55.4 & 35.0 & 44.0 \\
\hline 63 & $\alpha_{\mathrm{L}}$ & amide torsion & -1 & -26 & 60 & 51 & 48.9 & 49.0 & 43.9 & 32.7 & 47.3 & 64.3 & 38.5 & 50.7 & 51.0 \\
\hline 64 & $\alpha_{D}$ & $\mathrm{C} \alpha-\mathrm{CH}_{2} \mathrm{SH}$ torsion & 59 & 41 & 18 & -126 & 49.5 & 70.7 & 71.6 & 51.6 & 63.7 & 65.8 & 87.0 & 67.6 & 64.0 \\
\hline 65 & $\alpha_{D}$ & -SH rotation & 61 & 43 & -158 & 16 & 49.7 & 50.8 & 56.0 & 36.4 & 38.8 & 50.8 & 53.9 & 41.4 & 53.9 \\
\hline 66 & $\delta_{\mathrm{D}}$ & -SH rotation & -173 & -17 & 53 & 7 & 49.8 & 40.1 & 52.6 & 49.7 & 37.6 & 40.8 & 42.6 & 45.5 & 64.0 \\
\hline 67 & $\delta_{\mathrm{D}}$ & $\mathrm{C} \alpha-\mathrm{CH}_{2} \mathrm{SH}$ torsion & -152 & -51 & -12 & -58 & 50.6 & 38.4 & 42.5 & 47.5 & 37.2 & 46.8 & 52.3 & 51.4 & 57.5 \\
\hline 68 & $\delta_{\mathrm{D}}$ & $\mathrm{C} \alpha-\mathrm{CH}_{2} \mathrm{SH}$ torsion & -167 & -32 & 122 & 57 & 50.7 & 48.3 & 47.9 & 49.9 & 36.8 & 54.7 & 53.7 & 64.1 & 60.8 \\
\hline 69 & $\mathrm{C}_{7}^{\mathrm{ax}}$ & $\mathrm{C} \alpha-\mathrm{CH}_{2} \mathrm{SH}$ torsion & 58 & -38 & 20 & -77 & 50.7 & 44.8 & 45.9 & 36.0 & 42.0 & 57.3 & 58.5 & 57.7 & 64.6 \\
\hline 70 & $\mathrm{C}_{7}{ }^{\mathrm{eq}}$ & $\mathrm{C} \alpha-\mathrm{CH}_{2} \mathrm{SH}$ torsion & -80 & 99 & 118 & 162 & 50.8 & 51.9 & 64.1 & 54.9 & 43.6 & 66.8 & 70.0 & 59.9 & 42.5 \\
\hline 71 & $\delta_{\mathrm{D}}$ & -SH rotation & -113 & -62 & -57 & -122 & 52.5 & 52.0 & 49.5 & 40.5 & 40.3 & 59.2 & 67.5 & 45.6 & 54.6 \\
\hline 72 & $\alpha_{D}$ & amide torsion & 86 & 112 & -54 & -73 & 53.2 & 56.3 & 71.4 & 58.8 & 41.8 & 67.5 & 67.4 & 51.3 & 52.0 \\
\hline 73 & $\delta_{\mathrm{D}}$ & $\mathrm{C} \alpha-\mathrm{CH}_{2} \mathrm{SH}$ torsion & -180 & -33 & -112 & -94 & 53.3 & 47.5 & 68.6 & 57.0 & 39.0 & 58.3 & 64.3 & 70.6 & 66.8 \\
\hline 74 & $\delta_{\mathrm{D}}$ & $\mathrm{C} \alpha-\mathrm{CH}_{2} \mathrm{SH}$ torsion & -163 & -46 & 123 & 168 & 56.5 & 53.1 & 55.5 & 49.4 & 36.6 & 61.0 & 57.8 & 63.0 & 64.0 \\
\hline 75 & $\varepsilon_{\mathrm{D}}$ & $\mathrm{C} \alpha-\mathrm{CH}_{2} \mathrm{SH}$ torsion & 61 & -172 & 131 & 99 & 58.5 & 91.6 & 82.0 & 79.7 & 88.1 & 88.1 & 97.5 & 83.4 & 80.2 \\
\hline 76 & $\delta_{\mathrm{D}}$ & $\mathrm{C} \alpha-\mathrm{CH}_{2} \mathrm{SH}$ torsion & -133 & -56 & -16 & -169 & 60.3 & 54.4 & 56.5 & 48.8 & 43.3 & 64.1 & 65.2 & 55.5 & 63.1 \\
\hline 77 & $\delta_{\mathrm{D}}$ & -SH rotation & -151 & -45 & -101 & -72 & 61.3 & 45.9 & 56.3 & 50.3 & 39.5 & 56.4 & 60.0 & 58.9 & 69.6 \\
\hline \multicolumn{3}{|c|}{$\square \square \square \square$} & & & & & & 8.6 & 7.4 & 10.7 & 12.9 & 8.9 & 9.8 & 8.1 & 8.4 \\
\hline \multicolumn{3}{|l|}{$\mathbf{R}^{2}$} & & & & & & 0.72 & 0.78 & 0.56 & 0.70 & 0.77 & 0.74 & 0.79 & 0.86 \\
\hline
\end{tabular}


Journal of Chemical Theory and Computation 